\title{
Nota Terapéutica: Evaluando los medios de comunicación como fuente de información para el tratamiento farmacológico
}

Evaluating the Media as a source of Drug Therapy Information. Letter, issue 67, November - December 2007. Traducido con autorización de "Therapeutics Initiative" (The University of British Columbia). Disponible en URL: http://www.ti.ubc.ca/PDF/67.pdf

\author{
¿Qué sabemos de los reportes de prensa sobre terapia far- \\ macológica?
}

Los medios masivos de comunicación cumplen un rol primordial, informando al público y a los profesionales sobre nuevos descubrimientos y tratamientos ${ }^{1}$. Las historias relacionadas con temas de salud cada vez ocupan un espacio mayor en los medios. La mayoría de los diarios y estaciones de noticias tienen columnistas especializados en salud o programas dedicados a temas relacionados. Inclusive, las revistas médicas generan una cobertura masiva al emitir los comunicados de prensa de cada número². Estos mensajes de la prensa masiva influencian cada vez más el uso de las intervenciones sani$\operatorname{tarias}^{345}$. A pesar de esto, algunos investigadores cuestionan la calidad de los reportes de la prensa, y solicitan una aproximación más directa a las noticias de salud, y un reporte más completo de los resultados de las investigaciones ${ }^{689}$. Ver cuadro 1.

Cuadro 1: criterios propuestos por Media Doctor Canada para evaluar una noticia médica ${ }^{10}$
1. Tratamiento accesible
2. Tratamiento novedoso
3. Enfermedad inventada
4. Evidencia
5. Cuantificación de los beneficios del tratamiento
6. Cuantificación de los riesgos del tratamiento
7. Fuentes de información
8. Opciones terapéuticas
9. Costos del tratamiento
10. Confiabilidad de los reportes de prensa.

\section{¿Cómo se relacionan estos criterios con las notas de pren-} sa sobre Vitamina D?

El periodista reporta el caso de un estudio doble ciego aleatorizado, que siguió a 1179 mujeres postmenopáusicas sanas de un área rural de Nebraska, durante cuatro años. Las participantes fueron aleatorizadas a recibir $1500 \mathrm{mg}$ de calcio más $1100 \mathrm{UI}$ de Vitamina D3 (colecalciferol) o placebo ${ }^{11}$.

Accesibilidad: ¿El tratamiento en cuestión es accesible? El calcio y la Vitamina $D$ se encuentran muy accesibles en Canadá

Novedad: ¿Es verdaderamente un conocimiento nuevo o es otra mirada de lo que ya conocemos? ¿Este reportaje agrega nueva información a nuestro conocimiento del rol de la Vitamina D? La vitamina $D$ no es nueva y para entender qué agrega debemos evaluar la evidencia (ver más abajo).

Enfermedades inventadas: ¿Parece que están transformando una característica normal en una "enfermedad" o a una enfermedad aceptada la hacen parecer más amenazante o más prevalente de lo que realmente es? Nada obvio en este sentido parece haber con la Vitamina D.

Evidencia: ¿Cuál es la calidad de la evidencia por debajo de la cuestión de salud? La nota se basa en el mayor nivel de evidencia, un ensayo clínico aleatorizado.

Cuantificación del beneficio: ¿Cuáles son los beneficios relacionados con el tratamiento? Si se usan número relativos (por ej. reducción del $60 \%$ ) el periodista reporta el beneficio absoluto o cuántos pacientes, en relación a 100 con similar tratamiento, se beneficiarían (el número necesario a tratar)? El titular se refiere al beneficio relativo, pero adentrándose en la nota a uno le queda claro que el cálculo del beneficio relativo se basa en 50 pacientes con un cáncer nuevo: $4 \%$ en el grupo calcio, $3 \%$ en el grupo calcio mas vitamina D y $7 \%$ en el grupo placebo. De hecho, el uso concomitante de vitamina $D$ y calcio no reduce el riesgo de cáncer, cuando se lo compara con calcio sólo RR 0,76 (0,38 a 1,55) (cálculos propios).

Cuantificación del daño: ¿Cuáles son los riesgos del tratamiento? ¿Han sido cuantificados? No se mencionan los daños potenciales en la nota. No se reportan eventos adversos serios pero se mencionan cinco cálculos renales: tres en el grupo asignado a calcio, uno en el grupo asignado a calcio más vitamina $D$ y uno en el grupo placebo ${ }^{11}$.

Fuentes de información: ¿El periodista le dice si el investigador o el vocero citado en el artículo tiene algún lazo con la compañía que produce o comercializa el producto? No lo aclara.

Costo: ¿Cuál es el costo del tratamiento y cómo se compara con el costo de otros tratamientos que ya están disponibles? No se proveen datos. El costo diario del consumo de $1000 \mathrm{UI}$ de Vitamina D3 es alrededor de 0,07 dólares canadienses por tableta.

Opciones de tratamiento: ¿Existen otras opciones de tratamiento menos costosas o más seguras? Sentarse al sol es una fuente de vitamina $D$ no mencionada.

Confiabilidad de los reportes de prensa: ¿El periodista tomó información de reportes de prensa?: sí. ¿Tomó información de otras fuentes?: sí.

¿Qué dicen las últimas investigaciones sobre la calidad de los medios de comunicación en Canadá?

Media Doctor Canada analizó 87 historias relacionadas con tratamientos que aparecieron en importantes diarios de habla inglesa, un servicio de salud para profesionales y dos servicios de noticias por Internet, relacionados a dos cadenas de noticias (CBC y CTV) entre el 31 de Mayo de 2005 y el 1 de Noviembre de 2006. Los revisores utilizaron los criterios listados previamente para valorar la calidad de los reportes. La mayoría de las notar recibieron un "satisfactorio" en los ítems "tratamiento novedoso" $(93,8 \%)$ y enfermedad inventada $(89,7 \%)$. Tres criterios tuvieron un bajo puntaje: cuantificación de los riesgos del tratamiento $(8,2 \%)$, costos del tratamiento $(20 \%)$ y fuentes de Información (25\%).

¿Qué sucede con la información inadecuada que la prensa hace llegar a la gente?

Los periodistas reciben muy poca información relacionada con la calidad de lo que escriben, proviniendo ésta de sus editores, publicistas y muy pocas veces de sus lectores, a través de cartas al editor. Muy escasas veces los autores utilizan retroalimentación ("feed-back") profesional o auditoría, puntos considerados esenciales para asegurar la calidad de los reportes. Existen cuatro organismos que proveen feedback sobre la ca- 
lidad de los reportes en los medios de comunicación médica y realizan guías para los consumidores, que se detallan en el cuadro 2.

Cuadro 2: organismos anglosajones que auditan la calidad de los reportes en los medios de comunicación médica.

Australia: Media Doctor Australia (www.mediadoctor.org) Canadá: Media Doctor Canada (www.mediadoctor.ca) Estados Unidos: Health News Reviews (www.healthnewseviews.org)

Reino Unido: Hitting the Headlines (www.library.nhs.uk)

\section{¿Qué puede hacer un clínico cuando se encuentra con una historia en la prensa?}

Que una noticia sea precisa y completa puede prestar un valioso servicio público, colocando las expectativas de los consumidores en el lugar apropiado, o al contrario, promoviendo falsas expectativas sobre tratamientos de imprecisa efectividad, no basados aún en evidencia sólida ${ }^{4}$.Los profesionales de la salud deberían ser capaces de evaluar las noticias médicas para poder transmitirlas adecuadamente a sus pacientes, colocándolas en el contexto adecuado.

\section{¿Cómo le fue a la historia sobre vitamina $D$ en el puntaje de 10 ítems?}

Tuvo un puntaje de 5/10. Fue satisfactoria en los siguientes criterios:Tratamiento accesible, enfermedad inventada, tratamiento novedoso, cuantificación de los beneficios del tratamiento y opciones terapéuticas. El resto de los criterios fueron insatisfactorios.

\section{¿Debería esta historia y su ensayo clínico relacionado cambiar su práctica clínica?}

Cuando uno se da cuenta del escaso número de cánceres en este ensayo, y de que el beneficio principal proviene del calcio, uno se inclinaría a buscar resultados de trabajos más grandes.

\section{¿Existe alguna otra información de mejor calidad para informar a Ud. y a su paciente?}

La iniciativa de salud de las mujeres (en inglés: Women Health Initiave o WHI) es el más grande ensayo clínico relacionado a esta cuestión ${ }^{12}$. Comparó los efectos de la administración de $1 \mathrm{~g}$ de calcio y $400 U$ l de vitamina $D$ con el uso de placebo en más de 36000 mujeres seguidas durante siete años. EL WHI no demostró una reducción en el número de canceres asociados con calcio y vitamina D: RR 0,98 $(0,91$ a 1,05$)$. El reporte de prensa no nombró el WHI y el ensayo clínico en el cual se basó lo desestimó diciendo que se había utilizado una dosis inadecuada de vitamina $\mathrm{D}^{11}$.

\section{Conclusiones}

Los medios son importantes para diseminar nueva información médica por lo que la calidad y la completud de los reportes es vital. Muy frecuentemente los efectos terpéuticos no son ni tan espectaculares ni tan pobres como los reportan los medios. Los prefesionales de la salud deberían conocer cómo evaluar la calidad de la información proveniente de las noticias médicas.

El borrador de este informe fue remitido a 40 expertos y médicos de atención primaria para corregir cualquier inexactitud y asegurar que la información fuera concisa y relevante para los clínicos.

\section{Referencias}

1. Phillips DP, Kanter EJ, Bednarczyk B, Tastad PL. Importance of the lay press in the transmission of medical knowledge to the scientific community. New England Journal of Medicine 1991:325:1180-3.

2. Woloshin S, Schwartz LM. Press releases: translating research into news. JAMA 2002;287:2856-8.

3. Grilli R, Ramsay C, Minozzi S. Mass media interventions: effects on health services utilisation. Cochrane Database of Systematic Reviews 2002 (Issue 1).

4. Ries A, Ries L. The fall of advertising and the rise of PR. New York: HarperBusiness, 2002.

5. Basky G. Medical coverage of health stories often inaccurate, MDs report. CMAJ 1999;161:361

6. Entwistle V. Reporting research in medical journals and newspapers. BMJ 1995;310:920-3.

7. Schwartz LM, Woloshin S. The media matter: a call for straightforward medical reporting. Annals of Internal Medicine 2004;140:226-8.

8. Smith DE, Wilson AJ, Henry DA. Monitoring the quality of medical news reporting: early experience with media doctor. MJA 2005;183:190-3.

9. Cassels A, Hughes MA, Cole C, et al. Drugs in the news: an analysis of Canadian newspaper coverage of new prescription drugs. CMAJ 2003;168:1133-7.

10. Media Doctor Canada (www.mediadoctor.ca). see: rating information

11. Lappe JM, Travers-Gustafson D, Davies KM et al. Vitamin D and calcium supplementation reduces cancer risk: Results of a randomised trial. Am J Clin Nutr 2007:85:1586-1591.

12. Wactawski-Wende J, Kotchen JM, Anderson GL et al. Calcium plus Vitamin D supplementation and the risk of colorectal cancer. N Engl J Med 2006;354:684-696. 